\title{
Salt tolerance in wild relatives of adzuki bean, Vigna angularis (Willd.) Ohwi et Ohashi
}

\author{
Yutaro Yoshida $\cdot$ Rusama Marubodee $\cdot$ Eri Ogiso-Tanaka • \\ Kohtaro Iseki - Takehisa Isemura - Yu Takahashi - Chiaki Muto • \\ Ken Naito · Akito Kaga $\cdot$ Kazutoshi Okuno • Hiroshi Ehara • \\ Norihiko Tomooka
}

Received: 11 December 2014/ Accepted: 26 May 2015/Published online: 10 June 2015

(C) The Author(s) 2015. This article is published with open access at Springerlink.com

\begin{abstract}
Salt stress is becoming a serious problem in food production field. To find sources of salt tolerance, we screened 74 accessions of adzuki bean (Vigna angularis) and 145 accessions of cross-compatible wild relatives (seven species). We performed the primary screening in soil culture and the secondary screening in hydroponic culture, and identified JP205833 of $V$. riukiuensis (strain 'Tojinbaka') and JP107879 of V. nakashimae (strain 'Ukushima') as the valuable source of salt tolerance. We found these two strains had different salt tolerance mechanism, where
\end{abstract}

Yutaro Yoshida and Rusama Marubodee have contributed equally to this article.

Electronic supplementary material The online version of this article (doi:10.1007/s10722-015-0272-0) contains supplementary material, which is available to authorized users.

Y. Yoshida $\cdot$ K. Okuno

University of Tsukuba, Tennohdai 1-1-1, Tsukuba,

Ibaraki 305-8571, Japan

R. Marubodee $\cdot$ H. Ehara

Mie University, Kurimamachiya 1577, Tsu,

Mie 514-8507, Japan

E. Ogiso-Tanaka $\cdot$ K. Iseki $\cdot$ T. Isemura .

Y. Takahashi · C. Muto · K. Naito · A. Kaga .

N. Tomooka $(\bowtie)$

Genetic Resources Center, National Institute of

Agrobiological Sciences, Kannondai 2-1-2, Tsukuba,

Ibaraki 305-8602, Japan

e-mail: tomooka@affrc.go.jp
'Ukushima' prevented $\mathrm{Na}^{+}$accumulation in leaves by filtering $\mathrm{Na}^{+}$in roots and stems, while 'Tojinbaka' accumulated $\mathrm{Na}^{+}$throughout the whole plant body. We also found 'Tojinbaka' and 'Ukushima' could retain photosynthesis even under salt stress. In addition, 'Ukushima' and especially 'Tojinbaka' showed even better growth in a salt-damaged field in Fukushima, Japan where soybean cultivar 'Tachinagaha' could not survive. Since both salt tolerant strains are cross-compatible with adzuki bean, our results will facilitate developing salt tolerant cultivar by introducing two different mechanisms of salt tolerance.

Keywords Adzuki bean - Genetic resource - Genus Vigna $\cdot$ Legume $\cdot$ Salt tolerance $\cdot$ Wild crop relatives

\section{Introduction}

Soil salinity is a major constraint of food production because it limits crop yield and restricts uses of uncultivated land (Flowers and Yeo 1995). Currently, approximately $20 \%$ of irrigated cropland in the world has already been salt-damaged, and another $30 \%$ are considered salt-affected. In total, more than 800 Mha are salt-affected land in the world (FAO 2008). Given the rapidly growing world population, development of salt tolerant crop cultivars is inevitable to stabilize food production in cropland and to use currently unfavorable land. 
The genus Vigna (Leguminosae) comprises six subgenera distributed in Africa, Asia, Australia and America (Verdcourt 1970; Maréchal et al. 1978). Most of the Vigna species in Asia belong to the subgenus Ceratotropis, known as the Asian Vigna (Tomooka et al. 2002). Of these, six species have been domesticated; adzuki bean [V. angularis var. angularis (Willd.) Ohwi and Ohashi], mung bean [V. radiata (L.) Wilczek], black gram [V. mungo (L.) Hepper], moth bean [V. aconitifolia (Jacq.) Maréchal], rice bean [V. umbellata (Thunb.) Ohwi et Ohashi] and creole bean [V. reflexo-pilosa Hayata var. glabra (Maréchal, Mascherpa et Stainier) N. Tomooka et Maxted]. Wild relatives possess greater genetic diversity than their related cultigens, and have been adapted to various environments including coastal and saline areas (Tomooka et al. 2009). Therefore, such genetic resources are expected to contain genetic factors for salt tolerance, which might be useful for cross breeding (Shannon 1997).

In Asian Vigna species, adzuki bean, mung bean and black gram are especially important as human food. As for mung bean and black gram, belonging to section Ceratotropis, many studies of salt tolerance have been reported and some tolerant wild and cultivated accessions have been identified (Mohamed and El Kramany 2005; Sehrawat et al. 2013; Win et al. 2013). By contrast, for adzuki bean and other Asian wild species belonging to section Angulares, little has been studied (Yamauchi et al. 1989; Lee and Hong 2000). Adzuki bean is a traditional legume crop grown across East Asia and northern South Asia (Hanelt and IPK 2001; Zong et al. 2003). Since adzuki bean is cross-compatible with many wild species ( $V$. hirtella Ridley, $V$. minima (Roxb.) Owhi et Ohashi, $V$. nakashimae (Ohwi) Ohwi et Ohashi, V. nepalensis Tateishi et Maxted, V. riukiuensis (Ohwi) Ohwi et Ohashi, and $V$. tenuicaulis N. Tomooka et Maxted), (Doi et al. 2002) screening wild relatives for salt tolerance might be an efficient approach to develop salt tolerant cultivars via cross-breeding, and to isolate tolerance genes by linkage analysis.

Plant salt tolerance is considered to involve either avoidance ("excluder type") or tolerance ("includer type") mechanisms (Levitt 1980; Munns and Tester 2008). The "excluder type" plants are able to exclude toxic ions from internal plant tissue, while "includer type" plants take up $\mathrm{Na}^{+}$ions with a relatively lower toxicity (Johnson et al. 1991). In genus Vigna, only "excluder type" has been reported so far, where salt tolerant accessions of mung bean and $V$. unguiculata (L.) Walp. (cowpea) can restrict $\mathrm{Na}^{+}$uptake from the root or prevent $\mathrm{Na}^{+}$migration from the root to the aerial part (Jacoby 1964; Lessani and Marschner 1978; Fernandes de Melo et al. 1994; West and Francois 1982; Bernardo et al. 2006). In adzuki bean, similar mechanisms were reported in response to salt stress while no salt tolerant cultivars were found (Yamauchi et al. 1989).

In this study, we screened adzuki bean and its wild relatives for salt tolerance. Some of the selected strains were further investigated for $\mathrm{Na}^{+}$accumulation and photosynthetic response, and tested for growth in a salt-damaged field.

\section{Materials and methods}

Plant materials

A total of 219 accessions consist of eight Vigna species (including 149 accessions of seven wild species) were provided from the genebank in NIAS (National Institute of Agrobiological Sciences) in Tsukuba, Japan. See details in Table 1 and Electronic Supplementary Materials (ESM) Table 1.

Salt tolerance screening in soil culture

Primary screening for salt tolerance was conducted at NIAS in Tsukuba, Japan $\left(36^{\circ} 03^{\prime} \mathrm{N}, 140^{\circ} 10^{\prime} \mathrm{E}\right)$. The 219 accessions were grown in plastic pots $(7 \mathrm{~cm}$ in diameter $\times 9 \mathrm{~cm}$ in height) under natural light condition in a greenhouse from June in 2009. The soil used in the pot was KUREHA-Engei-Baido (KUREHA Inc, Japan). Twenty-one days after sowing, an adequate amount of salt solutions was applied in the pool and then the pots were immersed for 2 weeks. $\mathrm{NaCl}$ concentration was set to $0,50,100,150$ or $200 \mathrm{mM}$. Two plants with two replications were tested in each treatment.

Tolerance was evaluated at 1 and 2 weeks after treatment started. Each plant was visually scored by percent of wilted leaves as follows; $1=$ No leaves wilted, $2=1-25 \%$ of leaves wilted, $3=26-50 \%$ of leaves wilted, $4=51-75 \%$ of leaves wilted, $5=76-99 \%$ of leaves wilted and $6=$ all leaves wilted (ESM Fig. 1). 
Table 1 Plant materials used in this study

\begin{tabular}{lrl}
\hline Species & No. of accessions & Origin (no. of accessions) \\
\hline V. angularis & & \\
$\quad$ var. angularis & 69 & Japan (49), Republic of Korea (18), Nepal (2) \\
$\quad$ var. nipponensis & 5 & Japan (3), Myanmar (2) \\
V. hirtella & 31 & Thailand (5), Myanmar (7), Laos (19) \\
V. minima & 23 & Thailand (11), Indonesia (1), Myanmar (3), Laos (8) \\
V. nakashimae & 31 & Republic of Korea (30), Japan (1) \\
V. nepalensis & 1 & Nepal (1) \\
V. riukiuensis & 28 & Japan (27), Taiwan (1) \\
V. tenuicaulis & 30 & Thailand (4), Myanmar (24), Laos (2) \\
V. umbellata & 1 & Japan (1) \\
Total & 219 & \\
\hline
\end{tabular}

Salt tolerance screening in hydroponic culture

A total of 30 accessions from eight species (see Figs. 1, 2) was selected based on the result of primary screening (soil culture) and was sown in plastic pots with KUREHA-Engei-Baido supported and kept in green house for 21 days. Each accession was transplanted to a hydroponic culture in green house from August 2010. One culture pool $(62.5 \mathrm{~cm} \times 100 \mathrm{~cm} \times 17 \mathrm{~cm}$ height) contained 64 plants $(8 \times 8$ plants $)$. The culture pool contained diluted nutrient solution of a $1: 1$ ratio of Otsuka house No. 1 (1.5 g/L): Otsuka house No. 2 (1 g/ L) (Otsuka Chemical Co., Osaka, Japan: N, P, K, Ca and $\mathrm{Mg}=18.6,5.1,8.6,8.2$ and $3.0 \mathrm{mEq} / \mathrm{L}$, respectively). The nutrient stock solution was diluted to reach $\mathrm{EC}$ at $100 \mathrm{mS} / \mathrm{m}$ with water. Ten days after transplanting, salt stress was initiated. $\mathrm{NaCl}$ concentration in the hydroponic culture was $50 \mathrm{mM}$ for the first week, $100 \mathrm{mM}$ for the second week, and then $200 \mathrm{mM}$ from the third week to the eighth week. Wilt scores were evaluated every week after the initiation of salt stress.

Measuring $\mathrm{Na}^{+}$accumulation in plants

Four days after the $\mathrm{NaCl}$ concentration reached $200 \mathrm{mM}$, leaves, stems and roots were separately harvested from the three plants of $V$. angularis (JP233136, 'Kyoto Dainagon'), V. nakashimae (JP107879, 'Ukushima') and $V$. riukiuensis (JP235833, 'Tojinbaka'). The harvested samples were dried at $62{ }^{\circ} \mathrm{C}$ for $24 \mathrm{~h}$, ground into a powder and were reduced to ash for sodium extraction with $100 \mu \mathrm{L}$ of $1 \mathrm{~N} \mathrm{HNO}_{3}$. The cation concentrations in the leaves, stems and roots were determined using an ion chromatograph with a conductivity detector (Shimazu, Japan). Oxalic acid (3.3 $\mathrm{mM}$ ) was used as the mobile phase. The mobile phase was degassed by degasser (DGU-12A) and pumped with liquid chromatograph pump (LC-9A) at speed $1 \mu \mathrm{L} / \mathrm{min}$. This mobile phase was flown to the auto injector (SIL-6B) and mixed with $10 \mu \mathrm{L}$ of ample solutions to be homogenized, which was controlled by the system controller (SCL$6 \mathrm{~B})$. The $\mathrm{Na}^{+}$concentration was detected through the analytical column (IC-C3) in the column oven (CTO$10 \mathrm{~A})$ at $40{ }^{\circ} \mathrm{C}$. The result was printed by a chromatopac (C-R 6A). The standard solution of the $\mathrm{Na}^{+}$ concentration (for $100 \%$ was equated with $2 \mathrm{ppm}$ ) was measured for wring a standard calibration to calculate the correct ion concentrations of the sample solution.

Evaluation of photosynthetic activity and stomatal conductance

Seeds of $V$. angularis (JP233136, 'Kyoto Dainagon'), V. nakashimae (JP107879, 'Ukushima') and V. riukiuensis (JP235833, 'Tojinbaka') were germinated on Seramis (Effem GmbH, Verden, Germany) clay and hydroponically grown in a greenhouse for 4 weeks before measuring photosynthetic rate and stomatal conductance. To minimize the osmotic shock, $50 \mathrm{mM} \mathrm{NaCl}$ was added to the nutrient solution 3 days before the $\mathrm{NaCl}$ concentration was raised to $100 \mathrm{mM}$. The salt treated plants were grown for 7 days in $100 \mathrm{mM} \mathrm{NaCl}$ conditions. The control plants were grown in salt-free nutrient solution during the experiment. The photosynthetic rate and stomatal conductance of the topmost fully 
Fig. 1 Heat maps of wilt scores in the primary The 219 accessions were cultivated in soil culture and exposed to 50, 100, 150, or $200 \mathrm{mM}$ of $\mathrm{NaCl}$ for 1 week (left) and 2 weeks (right).

Wilt score of 1(no damage) is indicated as white whereas $6($ dead $)$ is indicated as strong orange. Accessions selected for secondary screening are indicated by arrows. Strains 'Ukushima' (JP107879), 'Tojinbaka' (JP235833) and cultivar 'Kyoto-Dainagon' (JP233136) are also indicated by longer arrows. (Color figure online) screening using soil culture.

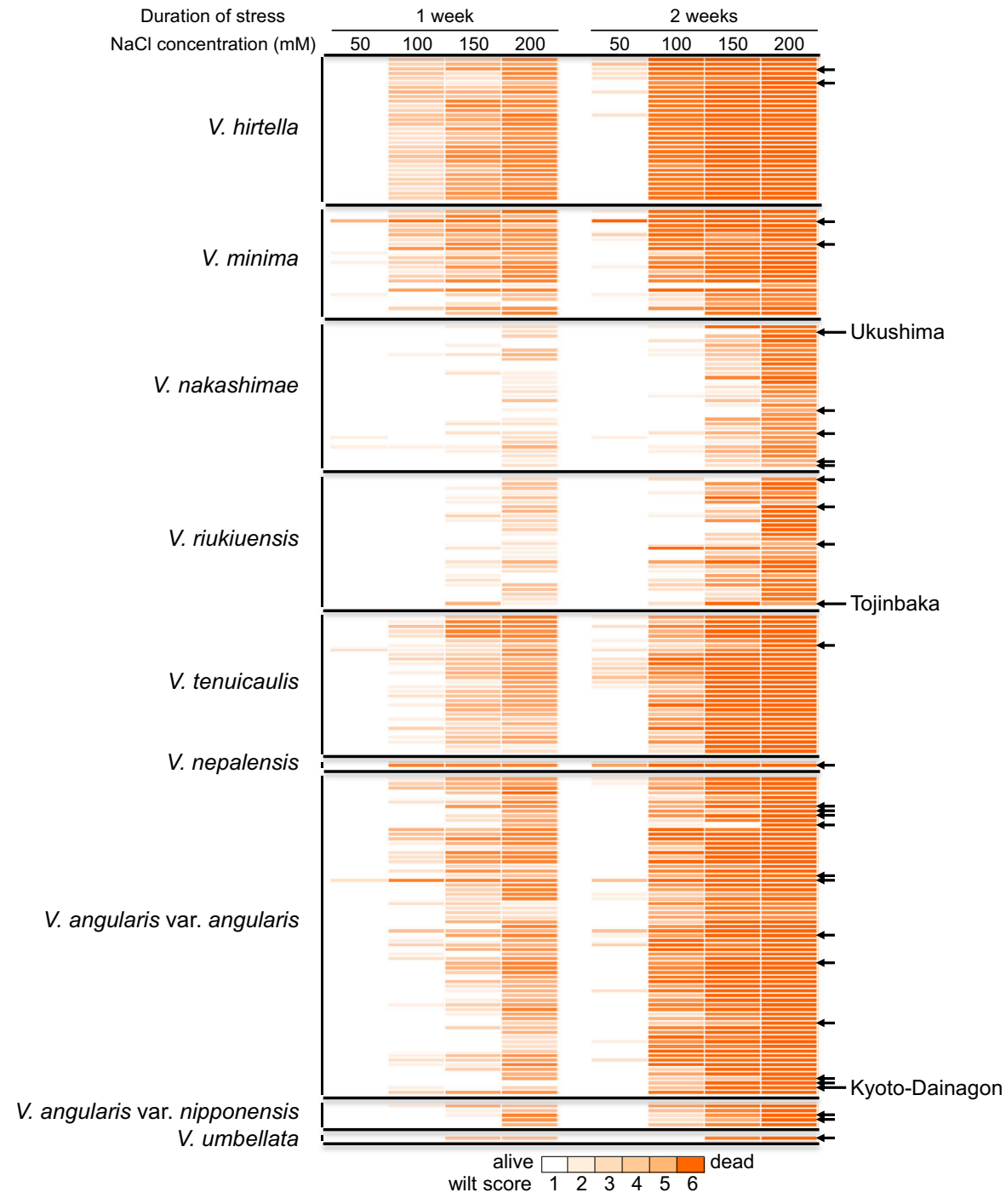

Growth test in a salt-damaged field

expanded leaves were measured using a portable photosynthesis system (LI-6400, LI-COR Inc., Lincoln, NE, USA). The measurements were obtained from 10:00 am-14:00 pm at 1 day before salt treatment, 3 days after $50 \mathrm{mM} \mathrm{NaCl}$ treatment, 3 and 7 days after $100 \mathrm{mM} \mathrm{NaCl}$ treatment. The measurement was done for 10 replications in each of salt treated and control plant under $1200 \mu \mathrm{mol}$ photon $\mathrm{m}^{-2} \mathrm{~s}^{-1}$ photosynthetic photon flux density, at $31.9 \pm 3.2{ }^{\circ} \mathrm{C}$ leaf temperature, $38.8 \pm 4.7 \%$ relative humidity and $399.9 \pm 0.2 \mathrm{ppm} \mathrm{CO}_{2}$ concentration. The measurements were performed after approximately $3 \mathrm{~min}$ of light exposure when the $\mathrm{CO}_{2}$ gas exchange rate reached a steady state.
We borrowed a salt-damaged field and a de-salted field from a farmer in Soma-city, Fukushima prefecture, Japan, where tsunami covered his field by the East Japan Earthquake on March 11, 2011. We sowed seeds of V.nakashimae (JP107879, 'Ukushima') and V. riukiuensis (JP235833, 'Tojinbaka') and soybean (Glycine max) (JP67666, cv. 'Tachinagaha') on June 11th, 2013, and harvested the whole plant shoot above ground on October 31th, 2013. We cultivated 32 plants for each strain/cultivar in a salt damaged and a deevaluate plant growth. salted field, and measured dry weight of each plant to 


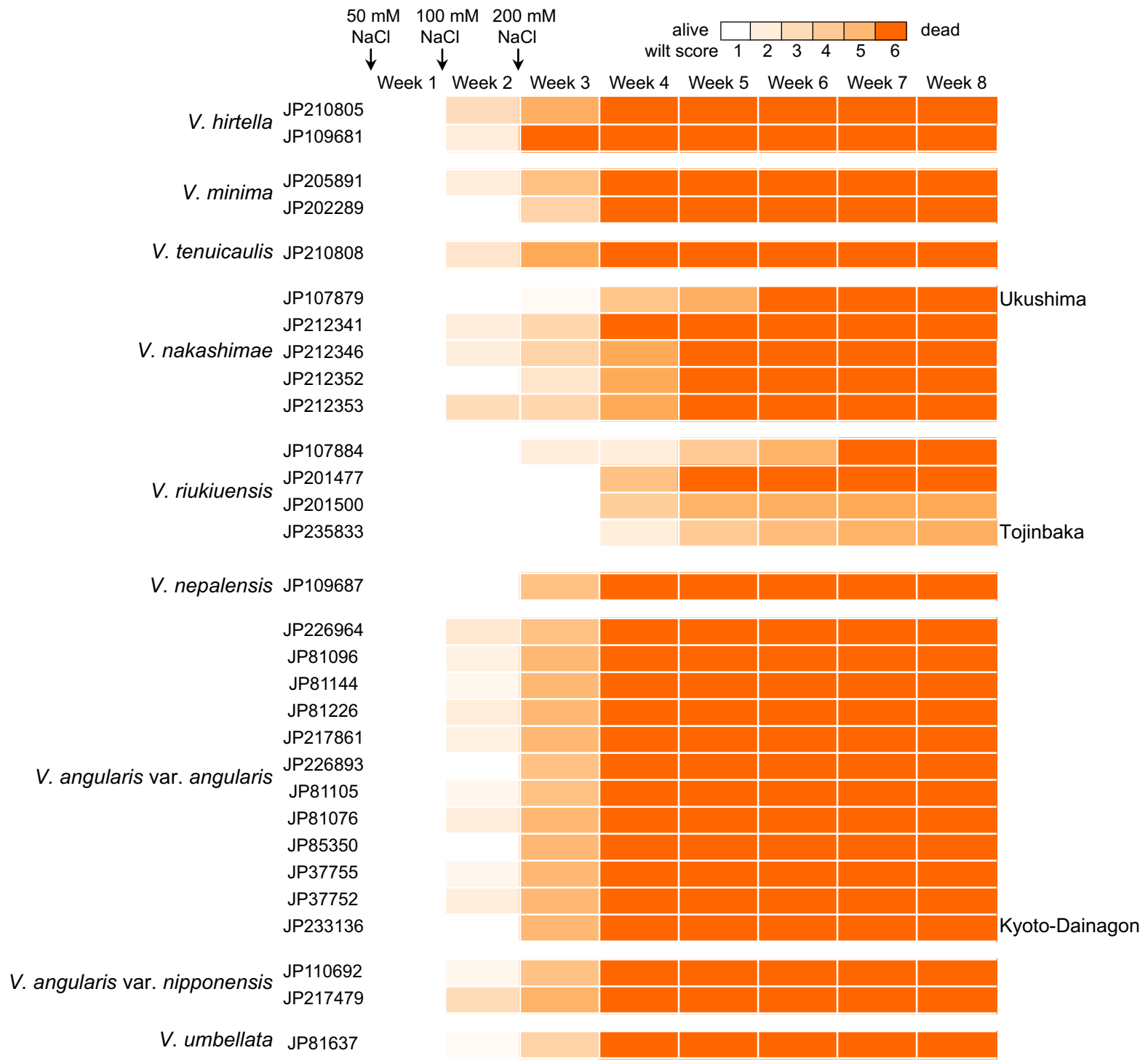

Fig. 2 Heat maps of wilt scores in the secondary screening using hydroponic culture. The 30 accessions selected from the primary screening were cultivated in a hydroponic culture with $\mathrm{NaCl}$. Wilt score of 1 (no damage) is indicated as white whereas

\section{Results}

Evaluation of salt tolerance in soil culture

A total of 219 accessions of eight species (Table 1, ESM Table 1) were evaluated for salt tolerance in soil culture (ESM Fig. 1; ESM Table 2). Levels of damage under $50,100,150$ or $200 \mathrm{mM}$ of $\mathrm{NaCl}$ for 1 and 2 weeks were visualized as heat maps based on the wilt score (Fig. 1). The results showed most
$6($ dead $)$ is indicated as strong orange. Times of onset $(50 \mathrm{mM})$ and increase of $\mathrm{NaCl}$ concentration $(100 \mathrm{mM}$ and $200 \mathrm{mM})$ are indicated at the top. (Color figure online)

accessions of $V$. nakashimae and $V$. riukiuensis were highly tolerant and survived 2 weeks in $150 \mathrm{mM}$ $\mathrm{NaCl}$. In this screening $V$. nakashimae showed better performance, since in $200 \mathrm{mM} \mathrm{NaCl}$ condition, 19 of 31 accessions of $V$. nakashimae survived 2 weeks, while only 3 of 28 accessions of $V$. riukiuensis could survive. Especially, JP212341, JP212342 and JP107879 (strain 'Ukushima') of $V$. nakashimae showed no visual damage symptoms in $150 \mathrm{mM} \mathrm{NaCl}$ for 2 weeks and in $200 \mathrm{mM}$ for 1 week (ESM 
Table 2). Large intraspecific variations were observed in V. minima, V.tenuicaulis and $V$. angularis, while no tolerant accessions were found in $V$. hirtella and $V$. napelensis. In cultivated accessions, one of the $V$. angularis accession (JP81144) was the most tolerant but leaf wilt was already observed at 2 weeks of $100 \mathrm{mM}$ treatment (ESM Table 2).

Evaluation of salt tolerance in hydroponic culture

According to soil culture screening, we selected 20 tolerant and 10 susceptible accessions and re-evaluated in hydroponic culture (Figs. 1, 2; ESM Table 3). To attenuate osmotic shock, we initiated salt stress with $50 \mathrm{mM} \mathrm{NaCl}$ for 1 week, $100 \mathrm{mM}$ for another 1 week, and finally set it to $200 \mathrm{mM}$. We evaluated wilt scores every week (ESM Fig. 1). The result was visualized as heat maps (Fig. 2). Almost all accessions were severely damaged at 1 week after $\mathrm{NaCl}$ concentration reached $200 \mathrm{mM}$ (Fig. 2; ESM Table 3). However, some accessions of $V$. nakashimae and $V$. riukiuensis exhibited no or little damage at this time point and survived for 2 weeks or longer in $200 \mathrm{mM}$ $\mathrm{NaCl}$ condition. In this experiment, JP235833, strain 'Tojinbaka' of $V$. riukiuensis, showed the highest tolerance and survived up to 6 weeks in $200 \mathrm{mM} \mathrm{NaCl}$ (week 8 in Fig. 2). Although V. nakashimae accessions scored the higher tolerance in soil culture screening, none of them could survive more than 3 weeks in $200 \mathrm{mM} \mathrm{NaCl}$ (week 6 in Fig. 2). Compared to soil culture, phenotypic variation in $V$. angularis and $V$. hirtella was smaller in hydroponic culture while that in $V$. nakashimae was greater.

$\mathrm{Na}^{+}$accumulation in plant body

According to the primary screening in soil culture and the secondary screening in hydroponic culture, we selected JP107879 of V. nakashimae (strain 'Ukushima') and JP235833 of $V$. riukiuensis (strain 'Tojinbaka'). To understand the mechanisms of salt tolerance in these strains, we measured the amount of $\mathrm{Na}^{+}$absorbed into the plants under salt stress. For comparison, we also tested JP233136 of V. angularis (cv. 'Kyoto-Dainagon'). During this experiment, we observed some chlorosis in the leaves of 'Ukushima', while most of the leaves of 'Tojinbaka' stayed green and maintained some growth (Fig. 3). In cv. 'KyotoDainagon' and strain 'Ukushima', $\mathrm{Na}^{+}$accumulation differed by tissues, where $\mathrm{Na}^{+}$amount was the least in the leaves and the highest in the stems ('Kyoto Dainagon', V. angularis) or in the roots ('Ukushima', V. nakashimae) (Fig. 4). However, in 'Tojinbaka' of $V$. riukiuensis, $\mathrm{Na}^{+}$accumulation was nearly the same between the tissues and thus the amount of $\mathrm{Na}^{+}$ accumulated in leaves was higher compared to cv. 'Kyoto-Dainagon' and strain 'Ukushima'.

Photosynthetic rate and stomatal conductance under salt stress

Vigna angularis cv. 'Kyoto-Dainagon' and $V$. nakashimae strain 'Ukushima' decreased Photosynthetic rate $(P n)$ rapidly after salt treatment (Fig. 5a). In contrast, in V. riukiuensis strain 'Tojinbaka', $P$ n did not decline under the condition of $50 \mathrm{mM} \mathrm{NaCl}$ (Fig. 5a). In $100 \mathrm{mM} \mathrm{NaCl}$, it started to decline but the extent of reduction was much smaller than that of cv. 'Kyoto-Dainagon' and strain 'Ukushima'. $P$ n value of 'Tojinbaka' at 13 days after salt stress onset was more than five times and two times

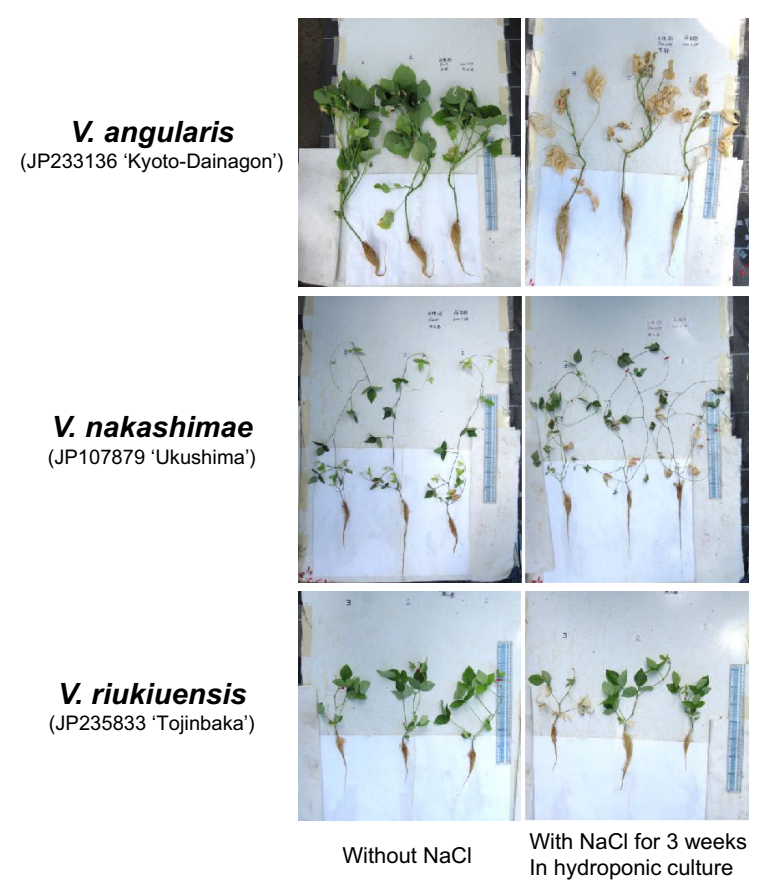

Fig. 3 Photos of adzuki bean cultivar 'Kyoto-Dainagon' (JP233136), strain 'Ukushima' (JP107879) of V. nakashimae and strain 'Tojinbaka' (JP235833) of $V$. riukiuensis grown in hydroponic culture under control (left) and salt-stressed conditions (right) for 16 days $(50 \mathrm{mM} \mathrm{NaCl}$ for a week, $100 \mathrm{mM}$ for another week and then $200 \mathrm{mM}$ ) 


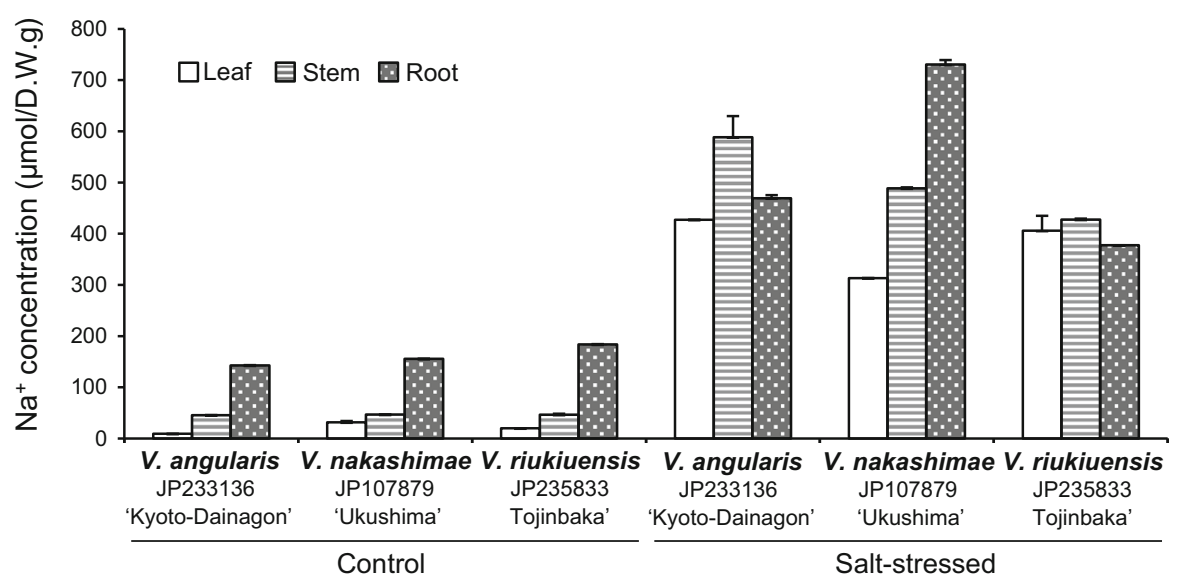

Fig. $4 \mathrm{Na}^{+}$concentrations in different plant tissues adzuki bean cultivar 'Kyoto-Dainagon' (JP233136), strain 'Ukushima' (JP107879) of V.nakashimae and strain 'Tojinbaka' (JP235833) of $V$. riukiuensis. Samples were collected 4 days after $200 \mathrm{mM}$

higher than that of 'Kyoto-Dainagon' and strain 'Ukushima', respectively.

Stomatal conductance (gs) of 'Kyoto-Dainagon', 'Ukushima' and 'Tojinbaka' under salt stress for 13 days or control condition was compared (Fig. 5b). The result showed that gs fell down to almost zero in 'Kyoto-Dainagon' and 'Ukushima' by salt stress, whereas 'Tojinbaka' still maintained gs approximately $25 \%$ of that in control condition.

Growth test in a salt-damaged field

Finally, we cultivated strain 'Ukushima' of $V$. nakashimae and strain 'Tojinbaka' of $V$. riukiuensis in a salt-damaged and de-salted farmer's field in Fukushima prefecture to evaluate salt tolerance in a field condition (Fig. 6a). For comparison, we cultivated soybean cv. 'Tachinagaha', which is one of the most popular soybean cultivars in this region. As a result, almost all the soybean plants were dead in the salt-damaged field, whereas 'Ukushima' of $V$. nakashimae showed comparable growth to that in the de-salted field, and, surprisingly, 'Tojinbaka' of $V$. riukiuensis showed even increased growth in the salt-damaged field (Fig. 6b).

\section{Discussions}

In this study, we have screened accessions of adzuki bean and the six related wild species for breeding salt
$\mathrm{NaCl}$ treatment (16 days after salt treatment). Leaf samples were collected including fallen leaves. Error bars indicate standard deviations $(n=3)$

tolerant cultivars or isolating tolerance genes. Our results revealed salt tolerance in adzuki bean was limited, but was prominent in several accessions of the two wild species, $V$. riukiuensis and $V$. nakashimae (Figs. 1, 2; ESM Tables 2, 3). Of these, we identified two strains, JP235833 of $V$. riukiuensis ('Tojinbaka') and JP107879 of $V$. nakashimae ('Ukushima') as highly valuable genetic source of salt tolerance (Figs. 1, 2, 3, 4, 5, 6). The amount of accumulated $\mathrm{Na}^{+}$in plant body revealed that the strain 'Ukushima' filtered $\mathrm{Na}^{+}$by roots and stems to prevent $\mathrm{Na}^{+}$uptake into leaves while 'Tojinbaka' accumulated similar amount of $\mathrm{Na}^{+}$throughout the whole plant body (Fig. 4). In addition, both strains have capacity to retain photosynthetic activity and growth under salt stress compared with that of adzuki bean cv. 'KyotoDainagon' or soybean cv. 'Tachinagaha', although this capacity is limited in 'Ukushima' (Figs. 5, 6).

Salt tolerance and other features in the selected strains

The different characteristics of $\mathrm{Na}^{+}$accumulation observed in strains 'Ukushima' and 'Tojinbaka' indicated different mechanisms of salt tolerance. Since in 'Ukushima' the amount of $\mathrm{Na}^{+}$uptake was highest in the roots and the lowest in the leaves, this strain is considered to have "excluder type" mechanism where $\mathrm{Na}^{+}$is excluded from internal tissue, or accumulated in apoplastic compartments (Levitt 1980, Fig. 4). In contrast, $\mathrm{Na}^{+}$was accumulated throughout 
$\mathbf{a}$

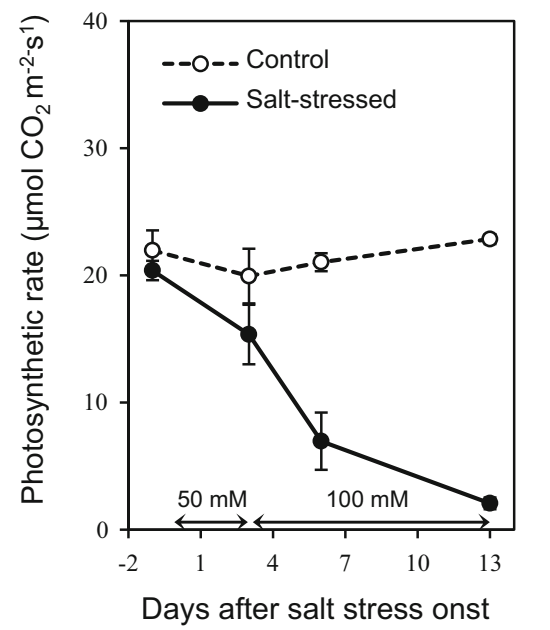

V. angularis

(JP233136 'Kyoto-Dainagon')

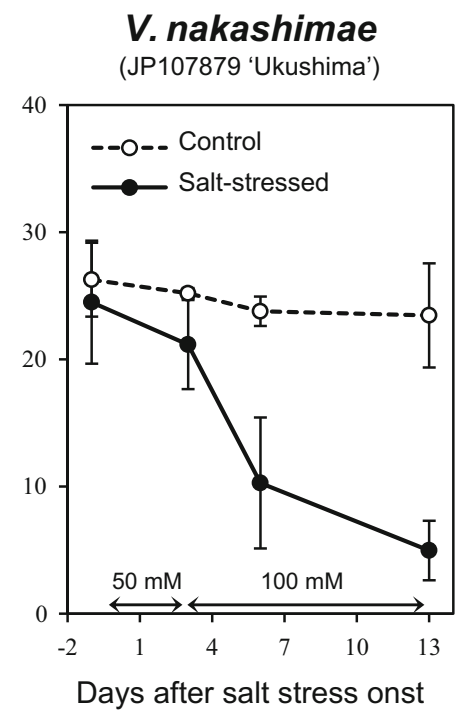

V. nakashimae

(JP107879 'Ukushima')
V. riukiuensis

(JP235833 'Tojinbaka')

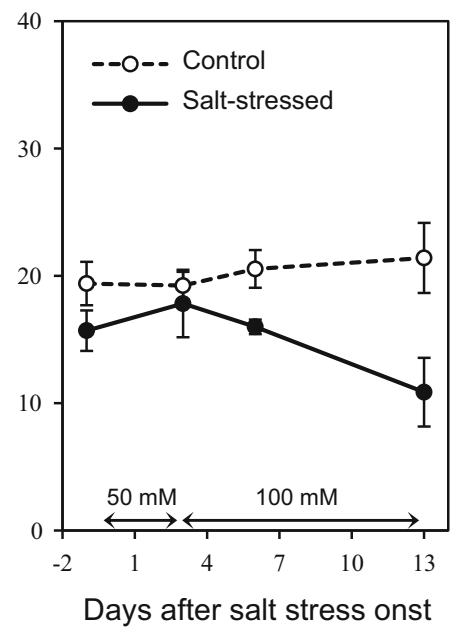

b

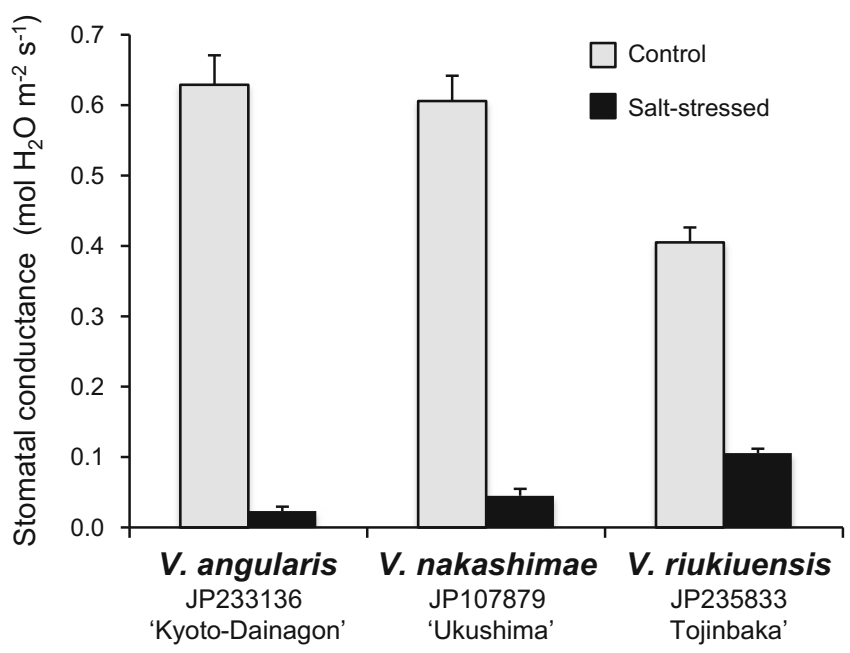

Fig. 5 a Photosynthetic rate $(P n)$ of the fully expanded leaves of control plants and salt stressed plants. $P$ n was measured 1 day before stress onset and 3, 6 and 13 days after $\mathrm{NaCl}$ treatment. The control plants were grown in salt-free nutrient solution during the experiment. $\mathrm{NaCl}$ treatment was started at $50 \mathrm{mM}$

the whole plant body in 'Tojinbaka', suggesting this strain has an "includer type" mechanism where $\mathrm{Na}^{+}$is partly detoxified or isolated into vacuoles (Levitt 1980). Perhaps the former mechanism was also present in adzuki bean cv. 'Kyoto-Dainagon' but was not strong enough to prevent $\mathrm{Na}^{+}$uptake into aerial parts, sine $\mathrm{Na}^{+}$uptake into the leaves were still lower compared to that into the stems (Fig. 4).

Since both 'Ukushima' and 'Tojinbaka' are cross compatible with adzuki bean, they could be of great
$\mathrm{NaCl}$ and raised to $100 \mathrm{mM}$ on the third day. Error bars indicate standard deviations $(\mathrm{n}=10)$. b Stomatal conductance $(g s)$ of fully expanded leaves of control plants and salt stressed plants on 13th day after salt stress treatment. Error bars indicate standard deviations $(\mathrm{n}=10)$

value for breeding to improve salt tolerance of this domesticated species. In addition, it would be interesting to introduce both "excluder type" and "include type" mechanisms into one adzuki bean cultivar. Further, crossing these two strains to adzuki bean would also facilitate linkage analysis to isolate genes of different salt tolerance mechanisms. Such genes, if identified, will be very useful to improve salt tolerance of crops through transgenic techniques, or genome editing. Indeed, we already developed $F_{2}$ plants 
Fig. 6 Field test of soybean (cv. 'Tachinagaha'),

V. nakashimae (strain

'Ukushima') and

V. riukiuensis (strain

'Tojinbaka') in a saltdamaged field. a Location of salt-damaged field, Somacity. b Dry weight of the plant shoots grown in a desalted field and a saltdamaged field $\mathbf{a}$

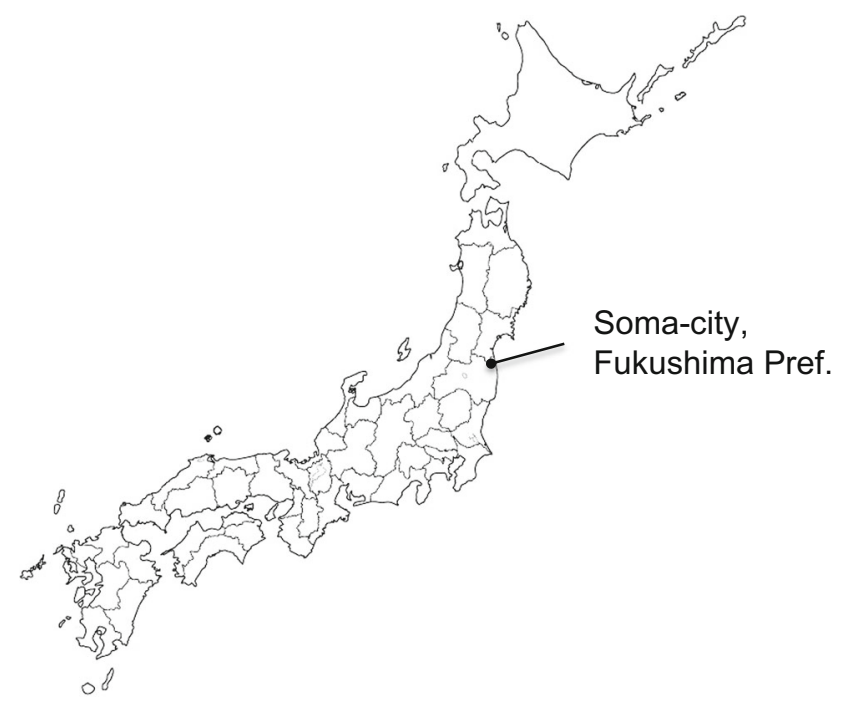

b

Soybean (cv. 'Tachinagaha')

V. nakashimae (JP107879 'Ukushima')

V. riukiuensis (JP235833 'Tojinbaka')
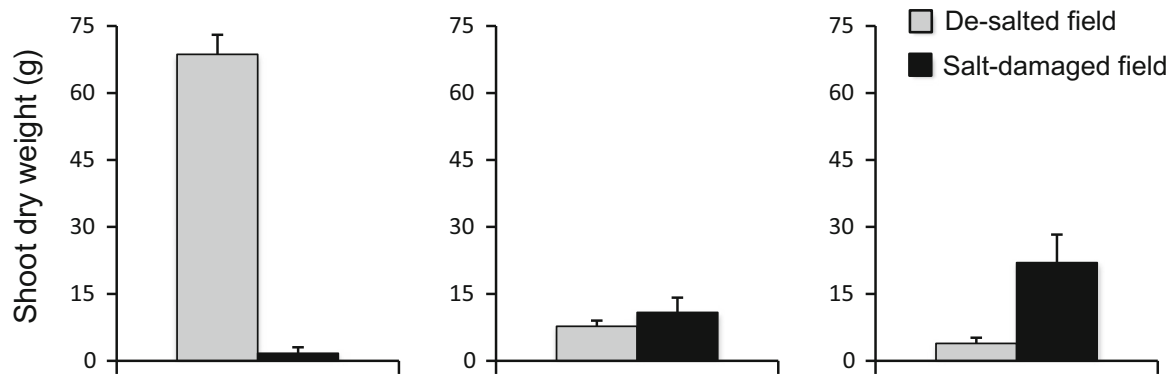

De-salted field

Salt-damaged field derived from 'Ukushima' $X$ 'Tojinbaka' and observed a transgressive segregation of salt tolerance (unpublished data). This fact suggested that salt tolerance in these two strains is genetically different and additive to each other.

Measuring photosynthetic response of 'Ukushima' and 'Tojinbaka' also revealed different features of these two strains. Even $50 \mathrm{mM}$ of $\mathrm{NaCl}$ decreased photosynthetic rate in adzuki bean cv. 'KyotoDainagon' (JP233136), but not at all in 'Tojinbaka' (Fig. 5a). In the $100 \mathrm{mM} \mathrm{NaCl}$ condition, cv. 'KyotoDainagon' almost lost stomatal conductance and arrested photosynthesis while 'Tojinbaka' still retained 50-60\% photosynthetic rate with relatively lower stomatal conductance (Fig. 5a, b). In 'Ukushima', the photosynthetic response to salt stress was similar to cv. 'Kyoto-Dainagon' but less severe. Thus, at least in hydroponic culture, the strain 'Tojinbaka' of $V$. riukiuensis is the most tolerant to salt stress. Interestingly, without salt stress, the strain 'Ukushima' exhibited higher photosynthetic rate than cv. 'KyotoDainagon', while 'Tojinbaka' achieved similar rate of photosynthesis with lower stomatal conductance (Fig. 5a, b). Thus, these two strains could also be useful in breeding for higher productivity (photosynthetic rate), drought tolerance or efficient water use (comparable photosynthetic rate under lower stomatal conductance).

In addition to the two strains described above, we also identified other tolerant accessions from $V$. nakashimae and $V$. riukiuensis. The tolerant accessions of these species are often found in coastal areas and thus are considered to adapt to salt affected environment (Tomooka et al. 1992, 2002). Our results 
also demonstrated the importance of detailed geographical information of genetic resources when screening for tolerance against environmental stresses.

\section{Peformance of selected strains under salt-damaged field}

The tsunami that followed the East Japan Earthquake on March 11, 2011 left 24,000 ha of salt-damaged farmland (Endo and Kang 2015). In the salt-damaged field, it is still impossible to cultivate crops including soybean (Fig. 6). However, both of the selected strains of $V$. nakashimae and $V$. riukiuensis, 'Ukushima' and 'Tojinbaka', respectively, showed similar or even better growth in the salt-damaged field. Therefore, these strains with different salt tolerance mechanism selected by soil pot and hydroponic culture experiments are considered to be practically useful genetic resources for breeding of salt tolerant adzuki bean cultivars.

Difference of salt tolerance in soil culture and hydroponic culture

Although several strains of $V$. nakashimae and $V$. riukiuensis repeatedly exhibited salt tolerance, we found some differences between the results of soil culture and hydroponic culture (Figs. 1, 2, 6; ESM Tables 2, 3). In general, strains of V. nakashimae were revealed to be more salt tolerant than those of $V$. riukiuensis in soil culture, whereas the tendency was opposite in hydroponic culture. Such differences might be because in soil culture the salt water in the pool gradually reached the root system of the plants, while in hydroponic culture plant roots were directly exposed to salt-containing solution. The effect of symbiotic arbuscular mycorrhizal fungi (Evelin et al. 2009) or other soil micro-organisms is also possible, which would make evaluation of salt tolerance more complicated. Thus, the screening in hydroponic culture might provide more direct evaluation of salt tolerance in genetic resources.

\section{Conclusions}

We would demonstrate that wild relatives of domesticated species are genetic resources of great value for tolerance against salt, and probably against other environmental stresses. The wild species of genus Vigna could be a good example according to its huge diversity of environmental habitations. Although we screened only $\sim 200$ wild accessions belonging to section Angulares, which is one of the sections of subgenus Ceratotropis of genus Vigna, we successfully obtained highly valuable source of salt tolerance. Since more diversity is found in other subgenera including subgenus Plectotropis and subgenus Vigna, we expect we can find more valuable source of stress tolerance. Finding adaptive traits and genes from the wild species will facilitate development of stress tolerant crops and contribute the issue of global food security.

Acknowledgments We appreciate the National Institute of Agrobiological Sciences (NIAS) genebank project for financial support and also supplying the seeds of accessions.

Open Access This article is distributed under the terms of the Creative Commons Attribution 4.0 International License (http:// creativecommons.org/licenses/by/4.0/), which permits unrestricted use, distribution, and reproduction in any medium, provided you give appropriate credit to the original author(s) and the source, provide a link to the Creative Commons license, and indicate if changes were made.

\section{References}

Bernardo MA, Enrique TD, José LGH, Raúl LA, Narciso YÁS, Sergio ZS, Edgar ORP, Cengiz K (2006) Effect of $\mathrm{NaCl}$ salinity in the genotypic variation of cowpea (Vigna unguiculata) during early vegetative growth. Sci Horticult 108:423-432

Doi K, Kaga A, Tomooka N, Vaughan DA (2002) Molecular phylogeny ofgenus Vigna subgenus Ceratotropis based on rDNA ITS and atpB-rbcL intergenic spacer region of cpDNA sequences. Genetica 114:129-145

Endo A, Kang DJ (2015) Salt removal from salt-damaged agricultural land using the scraping method combined with natural rainfall in the Tohoku district, Japan. Geod Reg 4:66-72

Evelin H, Kapoor R, Giri B (2009) Arvuscular mycorrhizal fungi in alleviation of salt stress: a review. Ann Bot 104:1263-1280

FAO (2008) Land and Plant Nutrition Management Service. http://www.fao.org/ag/agl/agll/spush

Fernandes de Melo D, Jolivet Y, Rocha AF, Gomes FE, Silva LM, Dizengremel P (1994) Effect of salt stress on mitocondrial energy metabolism of Vigna unguiculata cultivars differing in $\mathrm{NaCl}$ tolerance. Plant Physiol Biochem 32:405-412

Flowers TJ, Yeo AR (1995) Breeding for salinity resistance in crop plants-where next? Aust J Plant Physiol 22:875-884 
Hanelt P, Institute of Plant Genetics and Crop Plant Research (Eds.) (2001) Vigna angularis (Willd.) Ohwi \& Ohashi. In: Mansfeld's Encyclopedia of Agricultural and Horticultural Crops. 2: 753-754. Springer, Heidelberg

Jacoby B (1964) Function of bean roots and stems in sodium retention. Plant Physiol 39:445-449

Johnson SL, McPhee L, Birch LL (1991) Conditioned preferences: young children prefer flavors associated with high dietary fat. Physiol Behav 50:1245-1251

Lee HK, Hong JH (2000) Salt tolerance of Vigna angularis is during germination and early seeding growth. B Kor Envir Sci Soc 4:59-69

Lessani H, Marschner H (1978) Relation between salt tolerance and longdistance transport of sodium and chloride in various crop species. Aust J Plant Physiol 5:27-37

Levitt J (1980) Responses of plants to environmental stress. Volume II: water, radiation, salt, and other stresses. Academic Press, New York

Maréchal R, Mascherpa JM, Stainer F (1978) Etude taxonomique d'un groupe complexe d'espèces des genres Phaseolus et Vigna (Papilionaceae) sur la base de données morphologiques et polliniques, traitées par l'analyse informatique. Boissiera 28:1-273

Mohamed MH, El Kramany MF (2005) Salinity tolerance of some mung bean varieties. J Appl Sci Res 1:78-84

Munns R, Tester M (2008) Mechanisms of salinity tolerance. Ann Rev Plant Biol 59:651-681

Shannon MC (1997) Adaptation of plants to salinity. Ad Agron 60:76-120

Tomooka N, Egawa Y, Lairungreang C, Thavarasook C (1992) Collection of wild Ceratotropis species on the Nansei Archipelago, Japan and evaluation of bruchid resistance. JARQ 26(3):222-230
Tomooka N, Vaughan DA, Moss H, Maxted N (2002) The Asian Vigna: genus Vigna subgenus Ceratotropis genetic resources. Kluwer Academic Publishers, Dordrecht

Tomooka N, Kaga A, Isemura T, Vaughan DA, Srivines P, Somota P, Thadavong S, Bounphanousay C, Knyavong K, Inthapanya P, Pandiyan M, Senthil N, Ramamoorthi N, Jaiwal PK, Jing T, Umezawa K, Yokoyama T. (2009) Vigna genetic resources. The 14th NIAS international workshop on genetic resources, pp 11-21. http://www. gene.affrc.go.jp/pdf/misc/international-WS_14_11.pdf

Verdcourt B (1970) Studies in the Leguminosae-Papilionoideae for the flora of tropical East Africa: IV. Kew Bull 24:507-569

West DW, Francois LE (1982) Effects of salinity on germination, growth and yield of cowpea. Irrig Sci 3:169-175

Win KT, Oo AZ, Hirasawa T, Ookawa T, Hirata Y (2013) Genetic analysis of Myanmar Vigna species in responses to salt stress at the seedling stage. Afr J Biotechnol 10(9):1615-1624

Sehrawat N, Bhat KV, Sairam RK, Jaiwal PK (2013) Identification of salt resistant wild relatives of mung bean (Vigna radiata (L.) Wilczek). Asian J Plant Sci Res 3(5):41-49

Yamauchi M, Susaki S, Wakushima T, Fujyama H (1989) The mechanisms of salinity tolerance for adzuki bean (Phaseolus angularis L.) relationships between the varietal difference of salinity tolerance and characteristics of absorption and translocation of sodium ion. Jpn J Soil Sci Plant Nutr 60:325-334 [in Japanese with English summary (abstract)]

Zong XX, Kaga A, Tomooka N, Wang XW, Han OK, Vaughan DA (2003) The genetic diversity of the Vigna angularis complex in Asia. Genome 46:647-658 